\title{
OS EFEITOS DA IDENTIFICAÇÃO COM A MARCA DO FABRICANTE E DO ALINHAMENTO DO SISTEMA DE CONTROLE NAS VENDAS DO CANAL DE DISTRIBUIÇÃO
}

\begin{abstract}
Objetivo: Este trabalho tem por objetivos: (i) verificar o papel preditivo da identificação em explicar os resultados de vendas do fabricante no canal de distribuição, (ii) analisar os comportamentos positivos dos vendedores da revenda em relação à marca do fabricante e (iii) mensurar o efeito moderador do sistema de controle na relação entre identificação e resultados de vendas do fabricante.

Método: A população-alvo deste estudo foi 460 vendedores das 297 revendas de produtos e serviços de tecnologia da informação inscritas no programa de Parceria Direta da multinacional Alpha de computadores. No total, 147 questionários foram respondidos por esses profissionais de vendas

Originalidade/Relevância: A identificação organizacional criou maior resultado de venda para o fabricante quando há um maior alinhamento dos sistemas de controle de vendas, o qual é um moderador na relação.

Resultados: Os resultados demonstram que o sistema de controle do fabricante e a identificação com a marca aumentam significativamente os resultados de vendas do fabricante. Os achados também indicaram que a identificação com a marca gera comportamentos positivos do vendedor no canal de distribuição a favor da marca do fabricante.

Contribuições teóricas/metodológicas: $\mathrm{O}$ alinhamento do sistema de controle é positivo para os resultados de venda do fabricante porque significa que os diferentes agentes do processo de venda utilizam os mesmos sistemas de controle de resultados e de comportamentos dos vendedores, seja do fabricante como do canal de vendas e distribuição

Contribuições sociais / para a gestão: Quando a pressão dos procedimentos normativos e os incentivos financeiros direcionam os esforços do vendedor do canal de distribuição de uma determinada marca através do alinhamento dos sistemas de controle há um aumento de vendas. Reconhecendo isso, os fabricantes podem investir recursos para influenciar, de forma direta ou indireta, os sistemas de controle nos canais de distribuição. A aplicação do alinhamento dos sistemas de controle tende a melhorar o resultado do canal e elevar o desempenho dos membros.
\end{abstract}

Palavras-chave: Identificação. Sistema de Controle. Comportamento Positivo. Força de Venda. Canal. 


\title{
THE EFFECTS OF IDENTIFICATION WITH THE MANUFACTURER'S BRAND AND THE ALIGNMENT OF THE CONTROL SYSTEM ON DISTRIBUTION CHANNEL SALES
}

Objective: This paper addresses the manufacturer's efforts toward the distribution channel. Specifically, the research proposes that the channel's control system alignment with the manufacturer moderates the association between organizational identification with brand and performance, amplifying it.

Method: Using a survey of 147 salespeople from a multinational, the results demonstrated that the manufacturer's control system and organizational identification increased the manufacturer's sales.

Originality/Relevance: The organizational identification created greater revenues when there is a greater alignment of the sales control systems through the reinforcement of the psychological connection between brands and sellers in the distribution channel.

Results: The findings also indicated that the organizational identification with a brand generated positive behaviors of the seller in favor of the manufacturer brand.

Theoretical/Methodological Contrbutions: The alignment of the control system is positive for the manufacturer's sales results because it means that the different agents of the sales process use the same systems of control of results and behaviors of the vendors, be it the manufacturer or the sales and distribution channel.

Social / management contributions: When pressure from regulatory procedures and financial incentives drive the salesperson's efforts at the distribution channel of a particular brand through alignment of control systems there is increased sales. Recognizing this, manufacturers can invest resources to influence, directly or indirectly, control systems in distribution channels. Applying alignment of control systems tends to improve channel outcome and increase limb performance.

Keywords: Identification. Control System. Positive Behavior. Sales Force. Channel.

\author{
Rafael Artur Schuh ${ }^{1}$ \\ Cristiane Pizzutti ${ }^{2}$ \\ Valter Afonso Vieira ${ }^{3}$
}

\footnotetext{
${ }^{1}$ Mestre em Administração pela Universidade Federal do Rio Grande do Sul - UFRGS. Rio Grande do Sul, Brasil. Email: rafael.schuh@gmail.com

${ }^{2}$ Doutora em Administração pela Universidade Federal do Rio Grande do Sul - UFRGS. Professora da Universidade Federal do Rio Grande do Sul - UFRGS. Rio Grande do Sul, Brasil. E-mail: cristiane.pizzutti@ufrgs.br

${ }^{3}$ Doutor em Administração pelo Programa de Pós-Graduação em Administração da Universidade de Brasília - UnB. Professor na Universidade Estadual de Maringá -UEM. Paraná, Brasil. E-mail: vavieira@ uem.br
} 


\section{INTRODUÇÃO}

Os canais de distribuição em marketing exercem distintas funções no processo de desenvolvimento do produto, produção, armazenamento, distribuição intensiva ou seletiva por meio de distintos intermediários, alocação e entrega dos produtos facilitação aos consumidores finais (Berman, 1996; Wilkinson, 2001; Coughlan, Anderson, Stern, \& El-Ansary, 2006). Os canais de distribuição em marketing objetivam criar um diferencial competitivo, capaz de gerar sinergias e agregar valor a partir da redução dos custos e do aumento das vendas (Zhang, Liu \& Zhong, 2016).

A administração em busca de sinergias é tarefa importante para as empresas que utilizam canais indiretos de vendas, pois essas confiam a venda dos seus produtos aos membros do canal (ex. revendedores, corretores, agentes comerciais, atacadistas, varejistas). Nos canais de marketing existe um grande número de intermediários que desempenham uma variedade de funções (Mehta, Dubinsky \& Anderson, 2002; Almeida, Porto, Isidro, \& Coelho, 2014). O desafio para os fabricantes é como influenciar os intermediários que compõem o canal de distribuição para dedicarem recursos que possibilitem o atingimento das metas de vendas dos seus produtos (Coughlan et al., 2006). Esse desafio é notório, pois os intermediários representam uma ampla gama de fabricantes, de marcas e de produtos concorrentes nas mesmas categorias (Gale, 2005).

O fabricante por vezes não tem sistema de controle sobre o trabalho dos vendedores das revendas nos canais (Van Baal, 2014). Vendedores podem ter objetivos diferentes, tais como ter um melhor equilíbrio do portfólio de vendas das diferentes marcas e maximizar os ganhos. Para proteger o seu interesse, a revenda pode possuir um sistema de controles de vendas para direcionar o comportamento dos seus vendedores, pois o sistema de controles de vendas serve para alinhar objetivos e definir metas em conjunto entre o fabricante e o revendedor (Anderson, Lodish \& Weitz, 1987).

Fabricantes têm buscado implementar sistemas de controle com os distribuidores e os revendedores como instrumento de direcionamento dos esforços do canal de distribuição. Sistemas de controle de vendas representam uma série de procedimentos de monitoramento, direcionamento, avaliação e compensação financeira (Schmitz, Lee \& Lilien, 2014) para reduzir a ambiguidade das tarefas (Vieira, Rosa \& Faia, 2017) e os conflitos no dia a dia dos vendedores (Crosno \& Brown, 2015; Silva, Faia \& Vieira, 2016). Com base em Balduf, Cravens e Piercy (2005), os sistemas de controle tendem a ser uma combinação dos resultados das vendas (por exemplo, medidas de performance como $\mathrm{o}$ atingimento das metas definidas e crescimento das vendas) e dos resultados comportamentais (por exemplo, medidas de ações consideradas importantes para o atingimento desejado dos resultados como o número de visitas aos clientes e número de horas ao telefone com clientes).

Uma alternativa para melhorar o desempenho do canal de distribuição tanto para o fabricante quanto para o revendedor é via o aumento na identificação organizacional. A identificação tem o sentido de comprometimento de uma identidade (Bergami \& Bagozzi, 2000). Definida a partir das teorias de sociologia, psicologia e da identificação social (Tajfel \& Turner, 1985), a identificação tem impacto positivo nos diversos aspectos relacionados ao trabalho, tais como a satisfação no trabalho (Knippenberg \& Schie, 2000), a retenção de funcionários (Mael \& Ashforth, 1995), os comportamentos de cidadania organizacional (Meyer, Becker \& Van Dick, 2006), o comprometimento organizacional (Meyer et al., 2006), e o desempenho no trabalho (Ahearne, Bhattacharya \& Gruen, 2005). Adicionalmente, a identificação organizacional também tem sido pesquisada na relação entre identificação e marcas como, por exemplo, a identificação dos consumidores com empresas e a imagem das marcas (Bhattacharya \& Sem, 2003; Hildebrand, Fernandes, Veloso, \& Slongo, 2010). Contudo, pouco se sabe sobre a identificação do vendedor com a empresa e como tal identificação ajuda a marca a ser mais bem vendida no canal, gerando um problema dentro do canal de distribuição. Por consequência, o problema de pesquisa elaborado é: Qual é o papel da identificação e do comportamento positivo em explicar os resultados de vendas do fabricante no canal de distribuição dado a moderação do sistema de controle?

Utilizando o sistema de controle (Crosno \& Brown, 2015) para monitorar e alinhar diferentes canais de distribuição (Van Baal, 2014) e a identificação organizacional para observar o nível de congruência do vendedor com a marca, este trabalho tem por objetivos: $(i)$ verificar o papel preditivo da identificação em explicar os resultados de vendas do fabricante no canal de distribuição, (ii) analisar os comportamentos positivos dos vendedores da revenda em relação à marca do fabricante e (iii) mensurar o efeito moderador do sistema de controle na relação entre identificação e resultados de vendas do fabricante.

A partir de uma survey com 147 vendedores, este estudo explora o alinhamento dos sistemas de controle de vendas do canal de distribuição com o fabricante (chamado pelo nome fictício de empresa Alpha neste trabalho) e a identificação, e os seus impactos na performance de 
vendas do fabricante no mercado de computadores pessoais, o qual compreende os computadores de mesa (desktops) e os computadores portáveis (laptops). O mercado de computadores é caracterizado por múltiplos fabricantes tais como Dell, Sony, Compaq, Acer, Asus, etc., os quais competem pelos esforços que podem ser aplicados dos vendedores aos distintos produtos de seu portfólio (Yulinsky, 2000). Estes múltiplos canais de distribuição diferem em preço, promoções, variedade de produtos, conveniência e serviços (Goolsbee, 2001).

Esse trabalho está constituído da seguinte forma. O próximo tópico apresenta a fundamentação teórica e as hipóteses, abordando os conceitos principais dos sistemas de controle de vendas, da identificação e dos comportamentos positivos do vendedor. $\mathrm{O}$ próximo tópico destina-se à apresentação do método da pesquisa, incluindo a amostra, o instrumento de coleta de dados, e os procedimentos de análise dos mesmos. O próximo capítulo apresenta os resultados da pesquisa e as conclusões finais, limitações e sugestões para pesquisas futuras.

\section{BACKGROUND TEÓRICO}

\subsection{Sistema de Controle}

O sistema de controle de vendas engloba o monitoramento dos vendedores, o direcionamento das suas ações, as avaliações de performance e as atividades de reconhecimento dos vendedores e da equipe de vendas (Anderson \& Oliver, 1987; Murtha, Shervani, Challagalla, \& Kirkman, 2014). De acordo com O'Reilly (1989, p. 11), o sistema de controle gera "o conhecimento e objetiva dar atenção as ações realizadas pelos colaboradores para poder direcionar quando há possíveis desvios". Portanto, o sistema de controle requer atenção gerencial, padrões para comparações de resultados, e formas de identificar desvios, feedback e ações corretivas (Hofstede, 1978).

O alinhamento do sistema de controle significa que os gerentes, os vendedores, e os clientes utilizam os controles de resultados e de comportamentos, seja do fabricante ou do canal de vendas e distribuição, para buscar um ponto comum de concordância (Corcoran, Peterson, Baitch, \& Barrett, 1995). Dessa forma, o alinhamento do sistema de controle gerencial deve ser considerado um elemento estratégico para gerenciar a equipe de vendas e todos os sujeitos envolvidos (Faia \& Vieira, 2017). Os sistemas de controle de vendas podem ser classificados em aqueles que monitoram os resultados finais do processo ou aqueles que monitoram os comportamentos (Crosno \& Brown,
2015; Anderson \& Oliver, 1987; Silva, Vieira \& Faia 2015;).

Sistemas de controle de vendas baseados no resultado. Sistemas de controle de vendas baseados no resultado são sistemas em que os vendedores são livres para atingir os seus resultados, utilizando seus próprios meios e estratégias (Mullins, Ahearne, Lam, Hall, \& Boichuk, 2014). Nesse sistema, os vendedores são responsáveis pelos seus resultados, mas não são responsabilizados pelos métodos de atingimento. Historicamente, os gerentes de vendas e os sistemas de avaliação do desempenho de vendas tendem a alinhar os resultados das vendas para determinar as recompensas para os vendedores (Hochstein, Lilly \& Stanley, 2017; Churchill et al., 1979). Uma das principais razões desse alinhamento é o volume de vendas. O volume de vendas é uma medida muito popular em função do seu apelo intuitivo como uma medida de amplitude e profundidade (Weitz, 1981). Outras medidas baseadas nos resultados são margem bruta, margem líquida (vendas menos o custo do vendedor) e custo de vendas (Behrman \& Perreault, 1982).

Sistemas de controle de vendas baseados no comportamento. Os sistemas de controle de vendas baseado no comportamento endereçam o processo de venda (Miao \& Evens, 2013, 2014). Vendedores monitorados por esses sistemas podem ser avaliados e compensados quanto ao conhecimento do produto, qualidade de apresentação, capacidade de fechamento de vendas, serviços executados, número de contas ativas, chamadas realizadas, quantidade de correspondência e dias de trabalho (Jackson, Keith \& Schalcter, 1983). Portanto, o objetivo é alinhar os comportamentos necessários para o processo de vendas (Samaraweera \& Gelb, 2015).

A principal vantagem dos sistemas de controle baseados no comportamento é o controle que proporciona ao gerente (Kraus, Haumann, Ahearne, \& Wieseke, 2015). No sistema de controle por comportamento, os gestores podem direcionar os vendedores para executar certos comportamentos como parte da estratégia da empresa (Jaramillo, Bande \& Varela, 2015). Por exemplo, o compromisso de tempo investido pelo vendedor em previsão e em planejamento de vendas (Johnson, \& Sohi, 2017). Em suma, os sistemas baseados comportamentos permitem que as empresas possam executar estratégias com a força de vendas que envolvem trabalho de desenvolvimento e/ou certos comportamentos consistentes com a estratégia da empresa (Baldauf, Cravens, \& Piercy, 2005), gerando recompensa ou punição (Churchill et al., 1979). No sistema de controle por comportamento, Silva, Faia e Vieira (2016, p. 628) comentam que "os gestores se esforçam para controlar as tarefas dos vendedores que deverão ser executadas rotineiramente, nas quais eles podem intervir e dar 
suporte quando necessário, o que torna o controle mais intenso e frequente".

\subsection{Identificação Organizacional}

Baseada na teoria da congruência do self (SCT) de Sirgy (1982), a identificação organizacional é o quanto um funcionário identifica seus atributos como sendo congruentes aos atributos defendidos pela empresa em que trabalha (Tajfel \& Turner, 1985; Ashforth \& Mael, 1989). Um exemplo de como as organizações afetam seus membros através da identificação está nas palavras de um vendedor da $3 \mathrm{M}$ :

Eu descobri hoje que é muito mais fácil ser um vendedor da $3 \mathrm{M}$ do que de uma empresa qualquer que ninguém nunca ouviu falar. Quando você não tem que perder tempo justificando sua existência ou explicando por que você está aqui, dá-lhe uma certa quantidade de autoafirmação, além da receptividade calorosa e amigável (Garbett, 1988, p. 2).

Os vendedores possuem maiores níveis de identificação quando as organizações incorporam características pessoais. As características pessoais incorporadas pela organização transformam-se em padrões de comportamento que são utilizados pelos vendedores no contexto profissional e pessoal (Gecas, 1982; Markus \& Wurf, 1987).

O grau em que a identificação organizacional afeta a identificação de um vendedor pode depender da atratividade da imagem da organização (Dutton, Dukerich \& Harquail, 1994) para esta pessoa, e da avaliação subjetiva por parte do indivíduo (Kraus et al., 2015). Uma organização que possui uma identidade organizacional positiva pode ser mais atrativa para os vendedores (Lee, Park, $\&$ Koo, 2015). Dessa forma, pode-se presumir que quanto maior for a atratividade percebida em uma organização, mais forte poderá ser o vínculo entre as pessoas e a organização (Machado, 2003).

Teorias da identidade social afirmam que as pessoas procuram acentuar a sua própria diferenciação em contextos interpessoais (Tajfel \& Turner, 1985). Como resultado, os vendedores podem perceber as organizações como atrativas quando elas oferecerem alguma forma de diferenciação (ex. status, bônus, ganhos e prêmios) e identificação.

Ashforth e Mael (1989) argumentam que a identificação organizacional está associada à identificação cognitiva, na qual uma pessoa define a si mesmo com características similares à organização. No processo de definição e de aproximação, os funcionários podem acreditar que a organização possui características culturais únicas, uma estratégia diferenciada ou outros elementos que ajudam a empresa a se diferenciar no mercado.

A identificação dos vendedores também influencia o modo como eles acreditam que os demais sujeitos veem a organização (Lee, Park, \& Koo, 2015). A imagem externa construída atua como um espelho poderoso, refletindo aos funcionários como eles e a organização em que atuam são percebidos pela sociedade (Selame, 1988). Assim, a identificação se refere a impressão que uma organização passa aos membros internos e a comunidade com qual ela interage.

\section{HIPÓTESES}

A primeira hipótese sugere que o alinhamento dos sistemas de controle de vendas do fabricante e da revenda influencia positivamente nos resultados de vendas do fabricante (Oliver \& Anderson, 1994, Miao \& Evans, 2014). Em geral, os fabricantes esperam que as vendas da sua marca tenham um impacto positivo no desempenho de vendas global da revenda. No entanto, a revenda é responsável pela venda de um grande número de marcas - conhecido como multimarcas. Com o objetivo de reduzir a ambiguidade das decisões que são tomadas pelos vendedores da revenda quanto ao esforço no número de marcas, os fabricantes programam os sistemas de controle de vendas para maximizar seus resultados (Ahearne et al., 2010). Portanto, o alinhamento do sistema de controle de vendas entre fabricantes e revendedores tem como finalidade monitorar e controlar os fatores gerenciais, sistêmicos e de múltiplas marcas (Hughes \& Ahearne, 2010), gerando maior foco no resultado das metas, elevando as vendas. Por exemplo, há o controle no resultado das vendas e o controle no comportamento de esforço dos vendedores nas múltiplas marcas. Nesse sentido, os fabricantes no canal de distribuição utilizam os sistemas de controles alinhados e sistematizados com os objetivos do revendedor, influenciando assim o resultado de vendas. Logo, apresenta-se a primeira hipótese desta pesquisa:

$\mathbf{H}_{1}$ : O alinhamento dos sistemas de controle de vendas do fabricante e da revenda influencia positivamente nos resultados de vendas do fabricante.

A segunda hipótese pressupõe que a identificação do vendedor com a marca influencia positivamente os resultados de vendas do fabricante. Hughes e Ahearne (2010) apresentam a identificação do vendedor como uma tentativa menos formal de influenciar o desempenho canal de distribuição, pois o vendedor é propenso a agir de acordo com os 
grupos com os quais ele ou ela se identifica. Uma forte identificação com uma determinada marca ou com um grupo tende a dar origem aos desejos e tentações que apoiam o direcionamento da empresa, podendo assim elevar os resultados de vendas do fabricante (Ashforth \& Mael, 1989; Kraus et al., 2015). Por exemplo, o vendedor se identifica com o grupo de colegas que se esforça em vender especificamente uma marca dentre as marcas disponíveis, gerando maior congruência coletiva em prol do objetivo (Ashmore, Deaux \& MaclaughlinVolpe, 2004).

Além do mais, espera-se que exista uma relação positiva entre identificação com a marca e o desempenho de vendas do fabricante (Brown, Cron \& Slocum, 1997), pois quando o vendedor se identifica com a marca do fabricante, ações de apoio da organização são mais propensas de serem percebidas e implementadas pelo vendedor, resultando em um comportamento mais forte para as vendas (Brown \& Peterson, 1994). Essas ações de apoio podem ser por meio de treinamento, de suporte tecnológico e técnico, de comissão, entre outros.

H2: A identificação do vendedor com a marca influencia positivamente os resultados de vendas do fabricante.

A terceira hipótese sugere que o efeito direto da identificação do vendedor com a marca no resultado de vendas do fabricante é moderado pelo sistema de controle de vendas (Oliver \& Anderson, 1994). O efeito moderador do sistema de controle já foi examinado em outros estudos no que tange à inovação de produtos (Ahearne, Rapp, Hughes, \& Jindal, 2010; Vieira, Silva, Berbert, \& Faia 2015) e à orientação de locomoção de vendedores (Silva, Vieira \& Faia, 2015). Nós ampliamos esses achados para o campo da identificação organizacional.

Especificamente, se a identificação do vendedor com a marca é alta, os interesses e os objetivos do canal de distribuição tendem a priorizar às marcas do fabricante com maior nível de identificação (He, Wang, Zhu, \& Harris, 2015). Por consequência, se o sistema de controle de vendas monitorar e apoiar a marca de identificação do vendedor, é provável que o canal de distribuição aumente os esforços nas vendas relacionadas à marca em questão, amplificando os efeitos (Oliver \& Anderson, 1994). Nesse sentido, propõe-se que o sistema de controle de vendas tenha um papel moderador e amplifique $\mathrm{o}$ efeito direto da identificação do vendedor com a marca nas vendas do fabricante.

Por outro lado, se os sistemas de controle de vendas estão direcionados as outras marcas do portfólio do canal de distribuição (ex. multimarcas), o esforço do vendedor do canal de distribuidor é susceptível a diminuir (Hughes \& Ahearne, 2010), enfraquecendo a relação direta entre identificação e vendas. Dessa forma, apresenta-se a seguinte hipótese:

H3: O efeito direto da identificação do vendedor com a marca nos resultados de vendas do fabricante é moderado pelos sistemas de controle de vendas, de tal forma que quanto maior for alinhamento dos sistemas de controles de vendas, maior será este efeito.

$\mathrm{Na}$ próxima hipótese sugere-se que a identificação organizacional influencie comportamentos positivos em relação à marca do fabricante. Essa influência ocorre, pois a identificação organizacional tende a gerar um aumento na satisfação do trabalho, na redução da rotatividade dos funcionários e no engajamento das atividades de vendas, denominados comportamentos positivos (Ahearne, Bhattacharya, \& Gruen, 2005).

Assim como os empregados estão propensos a agir de acordo com os grupos e com as empresas que eles se identificam, o mesmo pode ocorrer com o vendedor do canal de distribuição (Bhatarchyya \& Sen, 2003). O vendedor está propenso a privilegiar a marca ou o distribuidor que ele possui maior identificação, o que influencia o seu comportamento. Além do mais, as metas que são relacionadas à identificação com a empresa, sejam elas metas com a marca ou com a empresa, direcionam um comportamento positivo e com isso incrementa os resultados de vendas (Hughes \& Ahearne, 2010). Portanto, formula-se a seguinte hipótese:

H4: A identificação do vendedor com a marca influencia positivamente os comportamentos positivos do vendedor ligados à marca do fabricante.

O comportamento positivo dos membros da organização está relacionado ao sentimento dos membros da organização em defender, apoiar e melhorar a organização com que se identificam (Mael \& Ashforth, 1995). Comportamentos positivos são ações proativas por parte do vendedor da revenda que estão fora do âmbito da descrição do seu cargo ou função, mas que contribuem para a viabilidade e a vitalidade da marca (Ahearne, Bhattacharya \& Gruen, 2005). Os comportamentos positivos podem gerar benefícios para o fabricante, cliente e vendedor, gerando assim resultados de venda do fabricante. Esse efeito benéfico do comportamento ocorre porque o vendedor pode promover a marca recomendando a mesma aos amigos, pode defender a marca de críticas, pode gerar relatórios de mercado que apontem os aspectos favoráveis da marca e pode, inclusive, corrigir 
situações de exposição negativa da marca (Hughes \& Ahearne, 2010). Como consequência:

H5: Comportamentos positivos do vendedor ligados à marca do fabricante influenciam positivamente os resultados de venda do fabricante.

O modelo teórico da Figura 1 apresenta o sistema de controle de vendas como influenciador direto dos resultados de vendas do fabricante e como moderador do efeito de identificação do vendedor com a marca. Ademais, o modelo teórico traz como consequência da identificação os comportamentos positivos do vendedor em relação à marca do fabricante (comportamento de suporte à marca) e o impacto desse último na venda do fabricante.

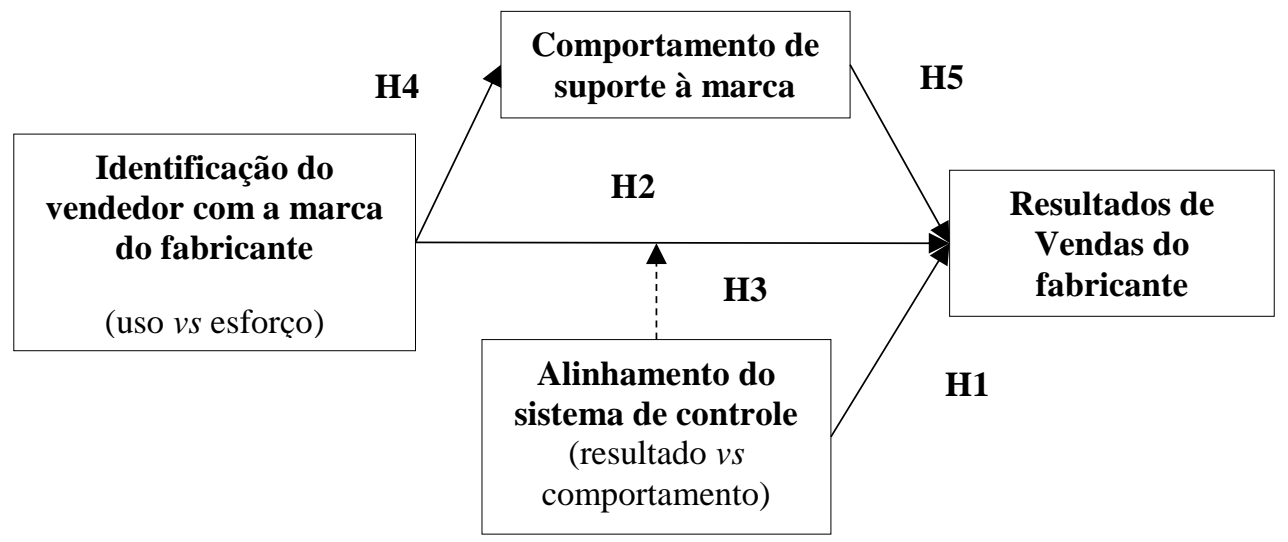

Figura 1 - Modelo teórico proposto

\section{MÉTODO}

\subsection{Contexto da Pesquisa}

A pesquisa quantitativa foi do tipo survey. A pesquisa foi aplicada com os revendedores da empresa Alpha, de computadores. A empresa Alpha está no mercado há mais de 30 anos com faturamento estimado de mais de 30 bilhões de dólares. Os vendedores são responsáveis por um volume de vendas anual que representa mais de $30 \%$ do faturamento total da multinacional Alpha no Brasil. Os vendedores vendem serviços de tecnologia da informação para organizações inscritas no programa de Parceria Direta, que é o programa de revendas da Alpha de computadores. O programa de Parceria Direta oferece financiamentos, acesso aos materiais de marketing, treinamentos e proteções de dados e sistemas.

Um questionário eletrônico foi criado e prétestado com os revendedores do canal. $\mathrm{O}$ mesmo foi testado em uma amostra de 12 vendedores de 6 revendas para verificar a clareza e o entendimento de todas as questões. Após ajustes necessários, a coleta de dados foi feita por meio da ferramenta Qualtrics. Os questionários foram enviados de forma online para as revendas no canal de distribuição (Apêndice I). Os vendedores das revendas acessavam o link e submetiam de volta as respotas.

\subsection{População-alvo}

A população-alvo deste estudo são quatrocentos e sessenta (460) vendedores das duzentos e noventa e sete (297) revendas de produtos e serviços de tecnologia da informação, inscritas no programa de Parceria Direta que é o programa de revendas da Alpha, as quais estão espalhadas por todo o território nacional. Esses vendedores são responsáveis por um volume de vendas anual que representa mais de $30 \%$ do faturamento total da Alpha no Brasil.

Cento e quarenta e sete (147) questionários foram respondidos $(31,96 \%$ de taxa de resposta) por profissionais de vendas, sendo que ao final, centro e trinta e nove (139) questionários foram aproveitados nesta pesquisa por apresentarem os dados completos para análise. Estas revendas que retornaram o questionário diferem em termos de tamanho, no entanto, todas atuam com as mesmas categorias de produtos e serviços no mercado de tecnologia da informação - revenda de hardware, software e serviços - para os seus respectivos mercados geográficos, portanto representam uma amostra significativa da totalidade das revendas cadastradas no programa de revendas da Alpha no Brasil. 


\subsection{Tratamento dos Dados}

Após a coleta e checagem dos dados não houve valores em banco na base de dados e, portanto, não houve necessidade de exclusão ou de substituição de valores. Na sequência, avaliou-se os valores extremos, os quais representam valores que são extremamente distantes da média. Pesquisadores possuem a opção de tratar os valores extremos com procedimentos sugeridos por Osbourne (2012), mas há a desvantagem de manipular a base de dados de modo artificial. Nesta pesquisa, nós optamos por deixar os valores extremos dos respondentes, pois são respostas oficiais dos revendedores da empresa Alpha.

\subsection{Amostra}

A população-alvo deste estudo foi 460 vendedores das 297 revendas de produtos e serviços de tecnologia da informação inscritas no programa de Parceria Direta da multinacional Alpha de computadores. No total, 147 questionários foram respondidos por esses profissionais de vendas (31,96\% de taxa de resposta), sendo que, ao final, 139 questionários (casos válidos) foram aproveitados nesta pesquisa por apresentarem os dados completos para análise.

As revendas que retornaram o questionário diferem em termos de tamanho. Contudo, todas atuam com as mesmas categorias de produtos e serviços no mercado de tecnologia da informação revenda de hardware, software e serviços - para os seus respectivos mercados. Como consequência, os vendedores representam uma amostra relevante da totalidade das revendas cadastradas no programa de revendas da Alpha no Brasil.

\subsection{Mensuração}

Segundo Anderson e Oliver (1987) e Oliver e Anderson (1994), o sistema de controle é composto por uma série de procedimentos para monitorar os funcionários e o alinhamento dos sistemas de controle de vendas refere-se ao grau em que esses diferentes sistemas monitoram, direcionam, avaliam e compensam comportamentos, atitudes e resultados semelhantes. Para mensurar o alinhamento do sistema de controle foi utilizada a escala de Hughes e Ahearne (2010). Foi solicitado aos vendedores do canal de distribuição que distribuíssem 100 pontos entre quatro marcas nomeadas, incluindo a marca do fabricante Alpha, e o quinto item sendo "outras marcas", como forma de identificar a ênfase que se coloca em cada marca.
Para medir a identificação do vendedor com a marca, utilizou-se a escala de Hughes e Ahearne (2010). Os vendedores foram solicitados a avaliar o grau de utilização da marca que vendem dentro e fora do trabalho, em ambientes ou em eventos sociais, e, ao mesmo tempo, até que ponto promovem a marca por vontade própria, considerando que a mesma possui atributos que geram um status social superior. Ademais, os vendedores da revenda foram solicitados a avaliar como se apresentam como profissionais da marca ou sentem orgulho de trabalhar com a marca do fabricante. Uma escala Likert de cinco pontos foi utilizada, variando de $1=$ nunca até $5=$ sempre.

Para a mensuração do comportamento positivo em relação à marca, utilizou-se a escala de Hughes e Ahearne (2010). Os vendedores foram questionados sobre até que ponto eles engajam em diferentes atividades voluntárias de promoção da marca, que vão além do escopo do seu trabalho. Por exemplo: o vendedor encoraja seus colegas à vender determinada marca do fabricante, arruma o material promocional que esteja fora do lugar, utiliza a marca e a recomenda a familiares e amigos, encoraja o seu gerente em fortalecer vínculos com a marca do fabricante, e informa $o$ fabricante sobre os movimentos da concorrência.

Os resultados de vendas do fabricante foram obtidos a partir dos registros de vendas existentes no sistema de gestão de vendas do fabricante com base na escala de Hughes e Ahearne (2010). Portanto, as vendas são registros de medição de performance de cada revenda. $\mathrm{O}$ método de mensuração foi similar ao utilizado por Ahearne, Jelinek e Jones (2007), com o objetivo de determinar a participação de um cliente no total de vendas de uma empresa e com isso determinar a sua relevância com cliente.

\section{RESULTADOS}

\subsection{Caracterização da Amostra}

A partir dos questionários aplicados, tendo como base as 297 revendas do programa de canais Parceiro Direto da empresa Alpha, 139 questionários foram considerados válidos. Questionários incompletos foram desconsiderados para a análise dos resultados. A predominância dos respondentes que possuem mais de 6 anos de tempo de experiência profissional no mercado foi de $51,8 \%$; sendo que $74,8 \%$ dos respondentes são homens (ver Tabela 1). 
Tabela 1 - Experiência da Revenda

\begin{tabular}{lcc}
\hline Níveis & $\begin{array}{c}\text { Experiência } \\
\text { Profissional }\end{array}$ & $\begin{array}{c}\text { Tempo de atuação na } \\
\text { Revenda }\end{array}$ \\
\hline Sem resposta & $17 \%$ & $8 \%$ \\
$<6$ meses & $3 \%$ & $13 \%$ \\
6 meses até 12 meses & $1 \%$ & $16 \%$ \\
$>12$ meses & $10 \%$ & $22 \%$ \\
$>36$ meses & $16 \%$ & $15 \%$ \\
$>60$ meses & $52 \%$ & $25 \%$ \\
\hline
\end{tabular}

Nota: $* * p<0,01$;

A Tabela 2 apresenta os dados sobre a faixa etária dos respondentes da pesquisa e mostra um equilíbrio na composição dos quadros de vendas das empresas respondentes de profissionais de jovens com 18 a 45 anos com a maioria das respostas (74,8\%), assim dividida: 18 a 25 anos, $21,6 \%$; 26 a 35 anos, $28,1 \%$ e 36 a 45 anos, $25,2 \%$.

Tabela 2 - Faixa etária dos respondentes

\begin{tabular}{lcc}
\hline Níveis & Frequência & Percentual \\
\hline Sem resposta & 11 & $7,9 \%$ \\
18 a 25 anos & 30 & $21,6 \%$ \\
26 a 35 anos & 39 & $28,1 \%$ \\
36 a 45 anos & 35 & $25,2 \%$ \\
46 a 55 anos & 13 & $9,4 \%$ \\
Mais de 55 anos & 11 & $7,9 \%$ \\
\hline
\end{tabular}

Nota: $* * p<0,01$;

Por fim, com o objetivo de caracterizar o grau de instrução dos respondentes, os respondentes foram questionados do seu grau de escolaridade. A distribuição das respostas encontram-se na Tabela 3 . De acordo com os resultados dessa tabela, pode-se dizer que grande parte dos respondentes $(41 \%$, soma dos respondentes com superior completo e pósgraduação) entende a necessidade de uma alta instrução como importante.

Tabela 3 - Escolaridade dos respondentes

\begin{tabular}{lcc}
\hline Níveis & Frequência & Percentual \\
\hline Sem resposta & 26 & $18,7 \%$ \\
Primeiro grau incompleto & 0 & $0,0 \%$ \\
Primeiro grau completo & 2 & $1,4 \%$ \\
Segundo grau incompleto & 5 & $3,6 \%$ \\
Segundo grau completo & 32 & $23,0 \%$ \\
Superior incompleto & 17 & $12,2 \%$ \\
Superior completo & 11 & $7,9 \%$ \\
Pós-graduação & 46 & $33,1 \%$ \\
\hline
\end{tabular}

Nota: $* * p<0,01$; 


\subsection{Análises Multivariadas}

A seguir foi desenvolvida a análise multivariada dos dados, envolvendo a modelagem em equações estruturais. Primeiramente, o modelo de medidas foi validado através da análise fatorial confirmatória, validade convergente e validade discriminante. Após isso, o modelo estrutural foi explorado com o objetivo de se verificar os coeficientes dos caminhos para a validação das hipóteses desta pesquisa.

A validade das escalas pode ser vista via confiabilidade composta e média da variância extraída (Henseler, Ringle, \& Sinkovics 2009). Os coeficientes de confiabilidade composta, no contexto da modelagem de equações estruturais, são indicados como substitutos dos coeficientes alfa de Cronbach e, à semelhança destes, retratam confiabilidade satisfatória quando apresentam valores acima de 0,70 (Henseler et al., 2009). Os valores obtidos de confiabilidade estão em concordância com estudos prévios (Santos \& Fernandes, 2008). A Tabela 4 apresenta a média dos construtos e os dados descritivos e o Apêndice II as cargas fatoriais.

Em seguida, analisamos a validade convergente que dá uma indicação do grau em que diferentes variáveis de medida de um mesmo construto estão correlacionadas e é verificada a partir da variância média extraída (AVE, da sigla em inglês para average variance extracted), cujos valores devem ser superiores a 0,50 (Henseler et al., 2009). Todos os valores da AVE estão acima do limite recomendado, o que permite afirmar que o modelo de mensuração possui validade discriminante.

Tabela 4 - Matriz de correlação dos construtos

\begin{tabular}{lcccccccc}
\hline Construto & Média & AVE & SD & $\begin{array}{c}\text { Confiabilidade } \\
\text { Composta }\end{array}$ & $\mathbf{1}$ & $\mathbf{2}$ & $\mathbf{3}$ & $\mathbf{4}$ \\
\hline 1 Comportamento Positivo & 3,80 & 0,67 & 1,18 & 0,92 & 1,00 & & \\
2 Vendas do fabricante & NA & 0,73 & NA & 0,94 & $0,23^{* *}$ & 1,00 & \\
3 Identificação & 3,80 & 0,54 & 1,22 & 0,91 & $0,73^{* *}$ & $0,33^{* *}$ & 1,00 \\
$\begin{array}{l}\text { 4 Alinhamento do Sistemas de } \\
\text { Controle }\end{array}$ & 70,00 & 0,84 & 32,53 & 0,98 & $0,56^{* *}$ & $0,30^{* *}$ & $0,58^{* *}$ & 1,00 \\
\hline
\end{tabular}

Nota: $* * p<0,01 ; \mathrm{NA}=$ não disponível; $\mathrm{SD}=$ desvio padrão

\subsection{Teste de Hipóteses}

A estimação do modelo estrutural foi feita com uso da modelagem de equação estrutural e com uso do software PLS (Hair, Gabriel \& Patel, 2014; Ringle, Silva \& Bido, 2014). A seguinte sequência foi adotada: (1) exame da magnitude dos coeficientes de determinação, (2) teste de significância e do sinal dos coeficientes, (3) verificação da moderação e (4) checagem do poder preditivo (Bagozzi, 1980; Henseler et al., 2009). Os resultados obtidos para os principais indicadores do modelo estrutural estão exibidos na Tabela 5 . Seguindo a recomendação de Henseler et al. (2009), verificou-se de início a significância dos coeficientes, por meio de um processo de reamostragem do tipo bootstrap e da análise da estatística $t$ de diferença de coeficiente. Para aplicar o bootstrap, optou-se por adotar 1000 repetições de amostras com reposição.

Os resultados mostraram que há um efeito direto do alinhamento do sistema de controle nos resultados de venda do fabricante $(\beta=0,21$; $p<0,001)$, suportando a $\mathrm{H}_{1}$. Portanto, nota-se que há relevância em alinhar os objetivos do fabricante com a revenda para obtenção de maior desempenho. Esse achado é esperado uma vez que os sistemas de controle de vendas ajudam a maximizar os resultados organizacionais (Ahearne et al., 2010; Crosno \& Brown, 2015)

Os dados indicaram que há um impacto positivo da identificação do vendedor com a marca nos resultados de venda dofabricante $(\beta=0,20$; $p<0,001)$, confirmando a $\mathrm{H}_{2}$. Assim sendo, o processo de se autoconectar com a organização ajuda o vendedor no sentimento de se pertencer, o que leva a uma melhora de seu desempenho. Esse resultado é congruente com os argumentos teóricos de que identificação gera congruência e esse matching ajuda a elevar resultados (Brown, Cron \& Slocum, 1997; Ashforth \& Mael, 1989; Kraus et al., 2015).

Por fim, a identificação do vendedor com a marca influenciou seu comportamento positivo para com a marca do fabricante $(\beta=0,83 ; p<0,001)$, conforme esperado na $\mathrm{H}_{4}$. A literatura mostra evidências de que o comportamento positivo eleva a satisfação do empregado (Ahearne, Bhattacharya \& Gruen, 2005) e com isso a marca pode receber benefícios. Em relação à $\mathrm{H}_{5}$, os resultados não suportaram o efeito direto do comportamento positivo do vendedor nas vendas do fabricante $(\beta=$ $0,59 ; p=\mathrm{NS})$. 
$\mathrm{Na}$ sequência, o efeito interativo da identificação com a marca e o alinhamento do sistema de controle foi verificado. Esse exame busca verificar a hipótese de moderação. Conforme o esperado, a identificação organizacional teve um impacto maior no desempenho do fabricante quando há alto valor de alinhamento dos sistemas de controle de vendas $(\beta=0,36 ; p<0,001)$. O resultado positivo e significativo mostra que o alinhamento do sistema de controle amplifica ainda mais o impacto direto da identificação do vendedor, o qual era de $\beta=0,20$ e com a moderação passa a ser de $\beta=0,36$.

Tabela 5 - Coeficientes da regressão do modelo estrutural

\begin{tabular}{llll}
\hline Relações Testadas & Beta & Sig. & Hipóteses \\
\hline H1 Alinhamento do sistema de controle ---> Vendas Fabricante & 0,21 & 0,001 & Suportada \\
H2 Identificação do vendedor ---> Vendas Fabricante & 0,20 & 0,001 & Suportada \\
H3 Identificação $\times$ sistema de controle ---> Vendas Fabricante & 0,36 & 0,001 & Suportada \\
H$_{4}$ Identificação do vendedor---> Comportamento Positivo & 0,83 & 0,001 & Suportada \\
H5 $_{5}$ Comportamento Positivo ---> Vendas Fabricante & $-0,59$ & 0,113 & Rejeitada \\
\hline
\end{tabular}

Conforme orientam Henseler et al. (2009), avaliou-se o impacto da moderação do alinhamento do sistema de controle de venda por meio do tamanho do efeito $\left(f^{2}\right)$. Para verificar a pertinência da moderação, utilizou-se a função de análise de variância $f^{2}$ do $\operatorname{SmartPLS}$, a qual foi executada para todas as variantes possíveis do modelo: sem a moderação e com a moderação (Hair, Sarstedt, Pieper, \& Ringle 2012; Hair, Gabriel \& Patel, 2014).
Os resultados obtidos para comparação dos modelos estão exibidos na Tabela 6 . Ao observar o resultado do modelo estrutural com a moderação, observa-se que há um coeficiente de determinação maior $\left(\mathrm{R}^{2}\right.$ moderação $=0,242)$ do que o modelo estrutural sem a

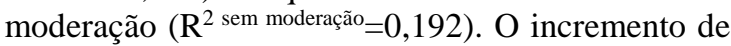
variância explicada é significativo e é conforme esperado.

Tabela 6 - Comparação dos modelos

\begin{tabular}{cccccc}
\hline Variante do Modelo & $\begin{array}{c}\text { Coeficientes de } \\
\text { Determinação }\left(\mathbf{R}^{2}\right) \\
\text { da Performance }\end{array}$ & $\begin{array}{c}\text { Significância } \\
\text { dos Caminhos }\end{array}$ & $\mathbf{f}^{\mathbf{2}}$ & $\begin{array}{c}\text { Interpretação } \\
\text { do Efeito }\end{array}$ & $\begin{array}{c}\text { Aceite da } \\
\text { hipótese }\end{array}$ \\
\hline Modelo sem moderação & 0,192 & $0,1 \%$ & & Sim \\
Modelo com moderação & 0,242 & $0,1 \%$ & 0,148 & $\begin{array}{c}\text { Efeito médio } \\
\mathrm{f}^{2} \sim 0,150\end{array}$ \\
\hline
\end{tabular}

Obs.: tamanho do efeito interpretado conforme Henseler et al. (2009, p. 304).

Por fim, a técnica de amostragens repetidas foi utilizada para avaliar a validade preditiva do modelo. Este método de avaliação é via blindfolding, em que se omite um caso por vez, estimando os parâmetros do modelo com o restante dos casos e predizendo os casos omissos (Vinzi, Chin, Henseler, \& Wang, 2010).

O blindfolding resulta no teste $Q 2$, conhecido como teste da relevância preditiva de
Stone-Geisser (Hair, Ringle \& Sarstedt, 2011). Se o valor de $Q 2$ for maior que zero, o modelo tem relevância preditiva e se for menor que zero falta relevância preditiva. Este teste foi aplicado utilizando-se a função "Blindfolding" do SmartPLS para se obter os valores Q2. Os valores estão exibidos na Tabela 7 e estão em conformidade com o esperado.

Tabela 7 - Teste Da Relevância Preditiva De Stone-Geisser

\begin{tabular}{lc}
\hline Construto & Q2 \\
\hline Alinhamento do sistema de controle & 0,80 \\
Identificação organizacional do vendedor & 0,40 \\
Comportamento Positivo com a marca & 0,59 \\
Vendas do fabricante & 0,58 \\
\hline
\end{tabular}




\section{CONCLUSÕES}

\subsection{Implicações Teóricas}

Os resultados do estudo demonstram que quanto maior for a identificação do vendedor do canal de distribuição com a marca, maior é o resultado de venda para o fabricante. Com isso, a identificação do vendedor do canal de distribuição, que é um fator psicológico, cognitivo e afetivo (Ashforth \& Mael, 1989), é um elemento que as empresas devem considerar para aumentar os esforços dos vendedores para aquelas marcas que geram maior identificação. A identificação do vendedor do canal de distribuição parte do pressuposto teórico que o vendedor pode se associar com a marca e/ou com o distribuidor.

Além do mais, os achados do presente estudo apresentam que a identificação do vendedor pode ser potencializada quando há um forte alinhamento no sistema de controle do canal de distribuição. O alinhamento é positivo para os resultados de venda do fabricante porque significa que os diferentes agentes do processo de venda utilizam os mesmos sistemas de controle de resultados e de comportamentos dos vendedores, seja do fabricante como do canal de vendas e distribuição (Corcoran et al., 1995). O construto alinhamento dos sistemas de controle e gestão de vendas considera o monitoramento dos vendedores, o direcionamento das suas ações, avaliações de performance e atividades de reconhecimento dos vendedores e dos gestores das equipes vendas (Anderson \& Oliver, 1987). Alinhamento significa que os diferentes agentes do processo de venda utilizam os mesmos sistemas de controle de resultados e de comportamentos dos vendedores, seja do fabricante como do canal de vendas e distribuição (Corcoran et al., 1995).

\subsection{Implicações Gerenciais}

$\mathrm{Na}$ pesquisa fica clara a relevância da gestão dos canais de distribuição como vantagem competitiva para o fabricante e melhora dos resultados da revenda (Van Baal, 2014). A multinacional Alpha recentemente mudou sua estratégia de distribuição e iniciou suas operações com canais indiretos para que também revendedores adquirissem produtos Alpha e os revendessem para clientes. A mudança de um modelo de distribuição unicamente direto da Alpha para um modelo híbrido com vendas diretas e canais indiretos é um claro exemplo da importância das estratégias de canal de distribuição como vantagem competitiva para as organizações.

Com base nos achados do trabalho, duas implicações gerenciais são oferecidas. Primeiro, quando a pressão dos procedimentos normativos e os incentivos financeiros direcionam os esforços do vendedor do canal de distribuição de uma determinada marca através do alinhamento dos sistemas de controle há um aumento de vendas. Reconhecendo isso, os fabricantes podem investir recursos para influenciar, de forma direta ou indireta, os sistemas de controle nos canais de distribuição. A aplicação do alinhamento dos sistemas de controle tende a melhorar o resultado do canal e elevar o desempenho dos membros.

Segundo, os fabricantes podem investir em mais suporte e geração de identificação organizacional com a marca para fortalecer $o$ sentimento de pertencimento dos membros do canal. Ao elevar a identificação organizacional, os vendedores obtêm maior desempenho para a marca do fabricante por um processo de auto categorização (Ashforth \& Mael, 1989). A identificação tem sido normalmente pesquisada no campo de marketing em estudos voltados à identificação com marcas em mercados de varejo ou em recursos humanos, aonde a identificação é instrumento de retenção dos talentos das empresas. No entanto, este estudo pesquisa as forças da identificação organizacional dentro do contexto de um canal de distribuição, testando a medida em que um vendedor do canal de distribuição se identifica com a marca do fabricante. Os resultados deste estudo demonstram que quanto maior for a identificação do vendedor do canal de distribuição, maiores serão os resultados para o fabricante.

\subsection{Limitações do Estudo e Sugestões para Futuras Pesquisas}

Em primeiro lugar, este estudo é por natureza de corte transversal de modo que apesar de termos toda cautela científica não há evidência estatística de causalidade (Bagozzi, 1980). Desta forma, futuras pesquisas podem superar esta limitação de causalidade da survey, incluindo outras metodologias. Estudos longitudinais, desenhos experimentais, estudo de caso e outras abordagens poderão oferecer contribuições valiosas a este tema de pesquisa.

Segundo, há uma limitação relativa à mensuração dos construtos em um único momento do tempo. Além de o fato exigir que os respondentes avaliem o evento retrospectivamente, as avaliações sobre um determinado aspecto do evento podem ser influenciadas por fatos ocorridos subsequentemente e podem contribuir para inflacionar as correlações entre os construtos e gerar maior influência das variáveis preditoras.

Terceiro, pesquisas futuras relacionadas ao canal de distribuição, com a introdução de novos 
moderadores, podem lançar luz sobre questões de como a identificação pode servir como substituto funcional para os sistemas de controle de vendas tradicionais. Por fim, neste estudo o fabricante e a marca pesquisada são os mesmos. Futuros trabalhos podem investigar a identificação em casos de fabricantes que possuem diferentes marcas, comparando os vendedores do canal de distribuição que se identificam com o fabricante de forma geral (ex. Sony) versus com alguma marca individual (ex. Vaio).

Quarto, pesquisas futuras poderiam examinar novos construtos no modelo. Por exemplo, a identificação organizacional (Kraus et al., 2015) pode ser analisada por meio da tensão organizacional, a qual é a percepção da identificação organizacional quando comparando gerentes do canal e vendedores. Espera-se que a tensão seja menor quando a congruência (Edwards 2002) entre gerentes do canal e vendedores elevando o desempenho (Kraus et al., 2015).

Quinto, pesquisas futuras poderiam examinar a liderança transacional e transformacional no modelo. É de conhecimento que a liderança transacional e a liderança transformacional possuem relações positivas com o desempenho (DeGroot, Kiker \& Cross, 2000; Judge \& Picollo, 2004) e são moderadas pelo sistema de controle (Kraus et al., 2015). Contudo, não se sabe como as lideranças podem ter um papel positivo no desempenho por meio do comportamento positivo.

\section{REFERÊNCIAS}

Ahearne, M., Bhattacharya, C.B. \& Gruen, T. (2005). Antecedents and Consequences of Customer-Company Identification: expanding $\mathrm{TH}$ Role of relationship marketing. Journal of Applied Psychology, 90(3), 574-585.

Ahearne, M., Jelinek, R. \& Jones, E. (2007). Examining the Effect of Salesperson Service Behavior in a Competitive Context. Journal of Academy of Marketing Science, 35 (4), 603-616.

Ahearne, M., Rapp, A., Hughes, D.E., \& Jindal, R. (2010). Managing sales force product perceptions and control systems in the success of new product introductions. Journal of Marketing Research, 47(4), 764-776.

Almeida, M. I. S., Porto, R.B., Isidro-Filho, A., Coelho, R.L.F. (2014). Impacto da extensão e interação de serviços nas vendas b2b: uma investigação temporal na categoria de combustíveis. REMark-Revista Brasileira de Marketing, 13(5), 124-139.
Anderson, E. \& Oliver, R.L. (1987). Perspectives on Behavior-Based Versus Outcome-Based Salesforce Control Systems. Journal of Marketing, 51(3), 76-88.

Anderson, E., Lodish, L.M. \& Weitz, B.A. (1987). Resource Allocation Behavior in Conventional Channels. Journal of Marketing Research, 24(1), 85-97.

Ashforth, B.E. \& Mael, F. (1989). Social Identity Theory and the Organization. Academy of Management Review, 14(1), 20-39.

Ashmore, R.D., Deaux, K. \& Maclaughlin-Volpe, T. (2004). An organizing framework for collective identity: Articulation and significance of multidimensionality. Psychological Bulletin, 130(1), 80-114.

Bagozzi, R.P. (1980). Causal models in Marketing. New York: John Wiley \& Sons.

Baldauf, A., Cravens, D.W. \& Piercy, N.F. (2005). Sales Management Control Research: synthesis and an agenda for future research. Journal of Personal Selling \& Sales Management, 25(1), 7-26.

Berman, B. (1996). Marketing Channels. Chichester: John Wiley \& Sons.

Behrman, D. N.; Perreault, W. D. (1982). Measuring the performance of industrial salespersons. Journal of Business Research, 10(3), 355-370.

Bergami, M. \& Bagozzi, R. P. (2000). SelfCategorization, Affective Commitment and Group Self-Esteem as Distinct Aspects of Social Identify in the Organization. British Journal of Social Psycho, 39(4), 555-577.

Bhattacharya, C. \& Sen, S. (2003). ConsumerCompany Identification: a framework for understanding consumers' relationships with companies. Journal of Marketing, 67(2), 76-88.

Brown, S. P. \& Peterson, R. A. (1994). The effect of effort on sales performance and job satisfaction. Journal of Marketing, 58(2), 70-80.

Brown, S. P., Cron, W. L., \& Slocum Jr, J. W. (1997). Effects of goal-directed emotions on salesperson volitions, behavior, and performance: A longitudinal study. Journal of Marketing, 61(1), 3950 . 
Churchill, G. (1979). A paradigm for developing better measures of marketing constructs. Journal of Marketing Research, 16(1), 64-73.

Corcoran, K. J., Peterson, L. K., Baitch, D.B. \& Barrett, M.F. (1995). High performance sales organizations. Chigado: Irwin.

Coughlan, A T., Anderson, E., Stern, L. W. \& ElAnsary, A I., (2006). Canais de marketing e distribuição. Porto Alegre: Bookman.

Crosno, J. L., \& Brown, J. R. (2015). A metaanalytic review of the effects of organizational control in marketing exchange relationships. Journal of the Academy of Marketing Science, 43(3), 297-314.

Dutton, J. E., Dukerich, J. M. \& Harquail, C.V. (1994), Organizational Images and Member Identification, Administrative Science Quarterly, 39(2), 239-263.

DeGroot, T., Kiker, D. S., \& Cross, T. C. (2000). A meta-analysis to review organizational outcomes related to charismatic leadership. Canadian Journal of Administrative Sciences, 17(4), 356-372.

Edwards, Jeffrey R. (2002), “Alternatives to Difference Scores: PolynomialRegression Analysis and Response Surface Methodology," In: Advances inMeasurement and Data Analysis, Drasgow Fritz and Schmitt Neil W., (eds). San Francisco, CA: Jossey-Bass, 350-400.

Faia, V.S. \& Vieira, V.A. (2017). Generating sales while providing service: The moderating effect of the control system on ambidextrous behavior. International Journal of Bank Marketing, 35(3), 447-471.

Gale, T. B. (2005). Shifts in Alcohol Distribution Channels. Modern Distribution Management, 35(14), 1-3.

Garbett, T. (1988). How to Build a Corporation's Identity and Project its Image. Lexington, MA: D. C. Heath.

Gecas, V., Turner, R.H. \& Short, Jr.J.F. (1982). The self-concept. Annual Review of Sociology, 8, 1-33.

Goolsbee, A. (2001). Competition in the Computer Industry: Online Versus Retail. Journal of Industrial Economics, 49(4), 487-499.
Hair, J. F., Ringle, C. M. \& Sarstedt, M. (2011). PLS-SEM: Indeed a silver bullet. Journal of Marketing Theory and Practice, 19(2), 139-152.

Hair, J.F., Gabriel, M.L.D.S. \& Patel, V.K. (2014). Modelagem de Equações Estruturais Baseada em Covariância (CB-SEM) com o AMOS: Orientações sobre a sua aplicação como uma Ferramenta de Pesquisa de Marketing. REMark-Revista Brasileira de Marketing, 13(2), 43-63.

Hair, J.F., Sarstedt, M., Pieper, T. M. \& Ringle, C. M. (2012). The use of partial least squares structural equation modeling in strategic management research: a review of past practices and recommendations for future applications. Long Range Planning Journal, 45 (5/6), 320-340.

He, H., Wang, W., Zhu, W. \& Harris, L. (2015). Service workers' job performance: The roles of personality traits, organizational identification, and customer orientation. European Journal of Marketing, 49(11/12), 1751-1776.

Henseler, J., Ringle, C. M. \& Sinkovics, R. R. (2009). The use of partial least squares path modeling in international marketing. In: Sinkovics, R. R. \& Ghauri, P. N. (Org.). New Challenges to International Marketing, Advances in International Marketing, 20, 277-319.

Hildebrand, D.F.N., Fernandes, V.D.H., Veloso, A.R. \& Slongo, L. A. (2010). Consumer-Company Identification: Development and Validation of a Scale. Brazilian Administration Review, 7(3), 276293.

Hochstein, B. W., Lilly, B., \& Stanley, S. M. (2017). Incorporating a Counterproductive Work Behavior Perspective into the Salesperson Deviance Literature: Intentionally Harmful Acts and Motivations for Sales Deviance. Journal of Marketing Theory and Practice, 25(1), 86-103.

Hofstede, G. (1978). The Poverty of Management Control Philosophy. Academy of Management Review, 3(3), 450-461.

Hughes, D. E. \& Ahearne, M. (2010). Energizing the Reseller's Sales Force: the power of brand identification. Journal of Marketing, 74(3), 81-96.

Jackson, D. W., Keith, J. E. \& Schlacter, J. L. (1983). Evaluation of selling performance: a study of current practices. Journal of Personal Selling and Sales Management, 3(4), 42-51. 
Jaramillo, F., Bande, B. \& Varela, J. (2015).

Servant leadership and ethics: A dyadic examination of supervisor behaviors and salesperson perceptions. Journal of Personal Selling \& Sales Management, 35(2), 108-124.

Johnson, J. S., \& Sohi, R. S. (2017). Getting business-to-business salespeople to implement strategies associated with introducing new products and services. Industrial Marketing Management, 62, 137-149.

Judge, T. A., \& Piccolo, R. F. (2004).

Transformational and transactional leadership: a meta-analytic test of their relative validity. Journal of Applied Psychology, 89(5), 755.

Knippenberg, D., \& Schie, E. (2000). Foci and correlates of organizational identification. Journal of Occupational and Organizational Psychology, 73(2), 137-147.

Kraus, F., Haumann, T., Ahearne, M. \& Wieseke, J. (2015). When sales managers and salespeople disagree in the appreciation for their firm: The phenomenon of organizational identification tension. Journal of Retailing, 91(3), 486-515.

Lee, E. S., Park, T. Y. \& Koo, B. (2015). Identifying organizational identification as a basis for attitudes and behaviors: A meta-analytic review. Psychological Bulletin, 141(5), 1049.

Machado, H.V. (2003). A identidade e o contexto organizacional: perspectivas de análise. Revista de Administração Contemporânea, 7(spe), 51-73.

Mael, F. A. \& Ashforth, B.E. (1995). Loyal from Day One: biodata, organizational identification, and turnover among newcomers. Personnel Psychology, 48(2), 309-333.

Markus, H. \& Wurf, E. (1987). The dynamic selfconcept: a social psychological perspective. In: M.R. Rosenweig \& L.W. Porter (Eds.), Annual Review of Psychology, 38, 299-337.

Mehta, R.; Dubinsky, A. I. \& Anderson, R. E. (2002). Marketing Channel Management and the Sales Manager. Industrial Marketing Managemen, . 31(5), 429-439.

Meyer, J. P., Becker, T. E., \& Van Dick, R., (2006). Social identities and Commitments at Work: Toward an Integrative Model, Journal of Organizational Behavior, 27(5), 665-683.
Miao C. F., \& Evans, K. R. (2013). The interactive effects of sales control systems on salesperson performance: a job demands-resources perspective. Journal of the Academy Marketing Science, 41(1), 73-90.

Miao, C. F. \& Evans, K. R. (2014). Motivating industrial salesforce with sales control systems: An interactive perspective. Journal of Business Research, 67(6), 1233-1242.

Mullins, R.R., Ahearne, M., Lam, S.K., Hall, Z.R. \& Boichuk, J. P. (2014, October). Know your customer: How salesperson perceptions of customer relationship quality form and influence account profitability. Journal of Marketing, 78(6), 38-58.

Murtha, B.R., Shervani, T. A., Challagalla, G.N. \& Kirkman, B.L. (2014). Control system diversity: Implications for selling centers. Journal of Business Research, 67(9), 1870-1876.

O'Reilly, C. (1989). Corporations, Culture and Commitment: Motivation and Social Control in Organizations. California Management Review, 31(4), 9-25.

Oliver, R. \& Anderson, E. (1994). An Empirical Test of the Consequences of Behavior- and Outcome-Based Sales Control Systems. Journal of Marketing, 58 (4), 53-67.

Osborne, J. W. (2012). Best practices in data cleaning: A complete guide to everything you need to do before and after collecting your data. Sage Publications.

Ringle, C.M., Da Silva, D. \& Bido, D.D.S. (2014). Modelagem de equações estruturais com utilização do SmartPLS. REMark-Revista Brasileira de Marketing, 13(2), 54-69.

Samaraweera, M. \& Gelb, B.D. (2015). Formal salesforce controls and revenue production: a metaanalysis. Journal of Personal Selling \& Sales Management, 35(1), 23-32.

Santos, C.P. \& Fernandes, D.V.H. (2008). A recuperação de serviços como ferramenta de relacionamento e seu impacto na confiança e lealdade dos clientes. RAE-Revista de Administração de Empresas, 48(1), 10-24.

Schmitz, C., Lee, Y. C. \& Lilien, G. L. (2014). Cross-Selling Performance in Complex Selling Contexts: An Examination of Supervisory-and Compensation-Based Controls. Journal of Marketing, 78(3), 1-19. 
Selame, J. (1988). The company image: building your identity and influence in the marketplace. Indiana University: John Wiley \& Sons.

Silva, J.D., Faia, V.S. \& Vieira, V.A. (2016). Os efeitos negativos da experiência e do controle no desempenho do vendedor. RAE-Revista de Administração de Empresas, 56(6), 626-640.

Silva, J.D., Vieira, V.A. \& Faia, V.S. (2015). Quando o sistema de controle enfraquece as vendas. REMark-Revista Brasileira de Marketing, 14(1), 115.

Sirgy, M. J. (1982). Self-Concept in Consumer Behavior: a critical review. Journal of Consumer Research, 9(3), 287-300.

Tajfel, H. \& Turner, J. C. (1985). The Social Identity Theory of Intergroup Behavior. In: Worchel, S.; Austin, W. G. (Eds.). Psychology of Intergroup relations. Chicago: Nelson-Hall, 7-24.

Van Baal, S. (2014). Should retailers harmonize marketing variables across their distribution channels? An investigation of cross-channel effects in multi-channel retailing. Journal of Retailing and Consumer Services, 21(6), 1038-1046.

Van Knippenberg, C.M. \& Van Schie, E.C.M.(2000). Foci and Correlates of Organizational Identification, Journal of Occupational \& Organizational Psychology, 73(2), 137-147, 2000.
Vieira, V.A., Rosa, M.A.G., \& Faia, V.S. (2017). The Mediating Role of Ambidextrous Salespeople in the Relationship between Stress and Performance. Revista de Administração Contemporânea, 21(2), 249-268.

Vieira, V.A., Silva, J.D., Berbert, S.C. \& Faia, V.S. (2015). Impacto do Sistema de Controle sobre a Venda de Novos Produtos. Revista de Administração Contemporânea, 19(2a ed. especial), 221-244.

Vinzi, V. E., Chin, W. W., Henseler, J., \& Wang, H. (Eds.). (2010). Handbook of partial least squares: Concepts, methods and applications. Springer Science \& Business Media: New York.

Weitz, B. A. (1981). Effectiveness in sales interactions: a contingency framework. Journal of Marketing, 45(1), 85-103.

Wilkinson, I. (2001). A History of Network and Channels Thinking in Maketing in the $20^{\text {th. }}$ Century. Australian Journal of Marketing, 9(2), 23-53.

Yulinsky, C. (2000). Multi-Channel Marketing: making bricks and clicks stick. Research Report. McKinsey Marketing Practice, Aug. 2000. Recuperado em 8 maio, 2014, de http://wggassociates.com/wp-content/uploads/pdfs/Mult ichannelmarketing,pdf.

Zhang, J., Liu, Z., \& Zhong, W. (2016). Attack and defend: the role of targeting in a distribution channel. Marketing Letters, 27(2), 375-386. 
ANEXO I - Escala de medidas

Alinhamento do Sistema de Controle de Vendas, Hughes e Ahearne (2010).

1. Programas de incentivo

2. Sistema de remuneração

3. Definição de objetivos de vendas

4. Determinação de ações de geração de demanda

5. Revisões periódicas de performance

6. Foco de discussões de vendas durante reuniões das equipes

7. Controle de vendas está alinhado com a organização

8. Calendário de vendas está alinhado com a organização

9. Foco geral

Identificação do vendedor com a marca do fabricante, Hughes e Ahearne (2010).

\section{Uso da Marca}

1. Pessoalmente utiliza a marca em casa

2. Pessoalmente utiliza a marca em locais públicos

3. Disponibiliza a marca para o uso de amigos ou a faz disponível em eventos sociais

\section{Esforço da marca}

4. Promoção da marca:

5. Se apresenta como vendedor da marca

6. Sente orgulho em trabalhar com a marca

7. Resolve problemas de outros em relação a marca

8. Sente responsável pelas ações da marca na sociedade

9. Se identifica com os valores da marca

Comportamento Extra da Marca Hughes e Ahearne (2010).

A partir de uma escala Likert de cinco pontos, sendo 1 "nunca", 2 "raramente", 3 "ocasionalmente", 4 "frequentemente" e 5 "sempre", os vendedores devem classificar as respostas a partir dos seus comportamentos individuais em até que ponto:

\section{Extra-role Brand Support}

1. Recomenda a marca para amigos

2. Defende a marca de críticas

3. Encoraja outros funcionários a vender a marca

4. Encoraja a gerência da revenda em fortalecer os vínculos com a marca

5. Informa a gerência da revenda as iniciativas competitivas da concorrência que impactam a marca

6. Corrigi situações como: falta de estoque; material de marketing com problemas; etc como forma de melhorar a promoção da marca na revenda

7. Comportamentos de apoio a marca:

8. Informa diretamente ao fabricante os movimentos da concorrência

Resultados de vendas do fabricante, Hughes e Ahearne (2010).

Os resultados de vendas do fabricante foram obtidos a partir de dados objetivos dos sistemas de vendas da Alpha do Brasil, para cada revenda participante nesta pesquisa:

1. Crescimento das vendas do fabricante, na revenda, nos últimos doze meses

2. Crescimento das vendas de produtos Enterprise (maior valor agregado), na revenda, nos últimos doze meses

3. Crescimento da margem do fabricante, na revenda, nos últimos doze meses 
4. Crescimento das vendas do fabricante, na revenda, nos últimos três meses

5. Crescimento das vendas de produtos Enterprise (maior valor agregado), na revenda, nos últimos três meses

6. Crescimento da margem do fabricante, na revenda, nos últimos três meses

\section{ANEXO II - Análise Fatorial das Escalas}

\begin{tabular}{|c|c|c|c|c|}
\hline \multirow{2}{*}{ Variáveis } & \multicolumn{4}{|c|}{ Cargas Fatoriais por Construto } \\
\hline & Sist Controle & Identificação & Comp Positivos & Performance \\
\hline Sist. Controle 1 & 0,92 & & & \\
\hline Sist. Controle 2 & 0,84 & & & \\
\hline Sist. Controle 3 & 0,94 & & & \\
\hline Sist. Controle 4 & 0,91 & & & \\
\hline Sist. Controle 5 & 0,95 & & & \\
\hline Sist. Controle 6 & 0,85 & & & \\
\hline Sist. Controle 7 & 0,89 & & & \\
\hline Sist. Controle 8 & 0,85 & & & \\
\hline Sist. Controle 9 & 0,98 & & & \\
\hline Identificação 1 & & 0,54 & & \\
\hline Identificação 2 & & 0,66 & & \\
\hline Identificação 3 & & 0,67 & & \\
\hline Identificação 4 & & 0,58 & & \\
\hline Identificação 5 & & 0,67 & & \\
\hline Identificacão 6 & & 0,42 & & \\
\hline Identificação 7 & & 0,77 & & \\
\hline Identificação 8 & & 0,88 & & \\
\hline Identificação 9 & & 0,75 & & \\
\hline Comp. Posit.1 & & & 0,81 & \\
\hline Comp. Posit.2 & & & 0,83 & \\
\hline Comp. Posit.3 & & & 0,91 & \\
\hline Comp. Posit.4 & & & 0,83 & \\
\hline Comp. Posit.5 & & & 0,85 & \\
\hline Comp. Posit.6 & & & 0,64 & \\
\hline Comp. Posit 7 & & & 0,61 & \\
\hline Vendas Fabric.1 & & & & 0,87 \\
\hline Vendas Fabric. 2 & & & & 0,55 \\
\hline Vendas Fabric. 3 & & & & 0,75 \\
\hline Vendas Fabric. 4 & & & & 0,76 \\
\hline Vendas Fabric. 5 & & & & 0,91 \\
\hline Vendas Fabric.6 & & & & 0,99 \\
\hline
\end{tabular}

